\title{
The role of logistic constraints in termite construction of chambers and tunnels
}

\author{
Dan Ladley, Seth Bullock \\ School of Computing, University of Leeds, Leeds LS29JT, UK
}

Received 1 October 2004; received in revised form 10 December 2004; accepted 10 December 2004

Available online 29 January 2005

\begin{abstract}
In previous models of the building behaviour of termites, physical and logistic constraints that limit the movement of termites and pheromones have been neglected. Here, we present an individual-based model of termite construction that includes idealized constraints on the diffusion of pheromones, the movement of termites, and the integrity of the architecture that they construct. The model allows us to explore the extent to which the results of previous idealized models (typically realised in one or two dimensions via a set of coupled partial differential equations) generalize to a physical, 3-D environment. Moreover we are able to investigate new processes and architectures that rely upon these features. We explore the role of stigmergic recruitment in pillar formation, wall building, and the construction of royal chambers, tunnels and intersections. In addition, for the first time, we demonstrate the way in which the physicality of partially built structures can help termites to achieve efficient tunnel structures and to establish and maintain entrances in royal chambers. As such we show that, in at least some cases, logistic constraints can be important or even necessary in order for termites to achieve efficient, effective constructions.
\end{abstract}

(C) 2005 Elsevier Ltd. All rights reserved.

Keywords: Stigmergy; Termite; Construction; Simulation; Model

\section{Introduction}

\subsection{Termites}

Social insects, such as termites, ants, wasps, and bees create some of the most spectacular structures seen in nature. Termite nests in particular are built on a scale only matched by that of human cities. Over and above their impressive scale, these architectures are also interesting because of their sophisticated functionality, containing purpose-built structures for fungus farming, air conditioning, etc. These achievements are all the more arresting when one considers that termite constructions are often built over many generations, and may require the cooperative enterprise of many millions of individual insects, each of which is in possession of only relatively simple mechanisms of communication

E-mail addresses: danl@comp.leeds.ac.uk (D. Ladley), seth@comp.leeds.ac.uk (S. Bullock). and control. We are certainly not yet in a position to be able to fully explain how simple termites manage to work together effectively to achieve such grand designs. However, what seems clear is that they cannot be relying upon some central source of executive control, either in the form of a "site foreman" (e.g. the queen termite) or some kind of explicit (genetic?) "blueprint".

Instead, the behaviour of individual termites appears to be driven by simple rules based on currently available pieces of local information such as temperature gradients, air flow, the presence or absence of partially complete structures, and, perhaps most importantly, the concentration of various pheromones that are excreted by the termites as they go about their work. What is critical here is that these environmental cues carry information about what activities have been carried out in the recent past-information that can profitably be used to guide current activity. Grassé (1984) uses the term stigmergy to describe this kind of indirect, environmentally mediated communication that 
is typically brought about as a side-effect of the very activity that requires coordination (Grassé, 1984). Though these stigmergic mechanisms are quite simple, it appears that through combining them in ways that are often subtle and complex, they can enable a colony to coordinate as a whole in order to construct complicated architectures. As the raw material from which nests are constructed is placed in the world, the local environment of passing builders is altered such that appropriate subsequent behaviours are encouraged. Structures that are incomplete "guide" workers to complete them.

For instance, "cement pheromone", which is given off by building material that has recently been deposited, is attractive to building termites, who approach sources of cement pheromone by climbing local cement pheromone intensity gradients. An additional tendency to deposit building material at locations where cement pheromone intensity is high brings about a positive feedback that increasingly focuses building effort on "hot-spots". One example of this kind of feedback-induced amplification of construction activity occurs at the outset of royal chamber construction. Royal chambers are enclosures built around a stationary queen termite (much larger than her builder relatives) who excretes a pheromone that encourages building activity at a certain level of intensity that will tend to occur some distance from the queen herself. Initially, building activity commences fitfully at a characteristic distance from the queen. Cement pheromone excreted by the first pieces of placed building material tends to attract nearby termites to these "proto-pillars" encouraging the contribution of additional building material to them. This results in the formation of a number of pillars that are distributed roughly evenly around the queen because pillars that are close together tend to "compete" for the attention of nearby termites, with one eventually winning out. This combination of negative and positive feedbacks results in evenly spaced pillars that are subsequently joined by low walls which rise until eventually a dome-like royal chamber with one or more entrances is completed. A third pheromone is deposited by moving termites and also guides building activity, encouraging the formation of galleries and covered walkways that protect heavily used thoroughfares. Even wind may affect the structures built by termites, who are thought to be sensitive to air currents and able to make decisions based on direct interaction with the wind (Bruinsma, 1979). In addition, any wind will disturb pheromone diffusion and may influence the structures being built as a result (Deneubourg et al., 1992; Bonabeau et al., 1997).

\subsection{Previous models}

The process of termite construction has been modelled several times (Deneubourg, 1977; Courtois and Heymans, 1991; Bonabeau et al., 1997). These models have mainly focused on the formation of pillars during the construction of Queen chambers. Deneubourg's mathematical model (1977), in particular, explained the regular spacing observed in nature as resulting from the positive feedback cycle involving cement pheromone emitted from recently placed building material, described above. A set of differential equations tracked the movement of termites, diffusion of cement pheromone and location of "active" (recently placed) building material over a 2-D world. From an initially random distribution of active material "pillars" could form through (i) the amplification of any initial variability and (ii) nearby pillars "competing" for termite attention.

This model was later expanded by Bonabeau et al. (1997) to include equations representing factors such as wind, a pheromone template emitted by a stationary queen, and an artificially imposed net flow of termites across the world. The queen pheromone template allowed the structure of royal chambers to be modelled. A net flow of termites led to the construction of walkways and, where these walkways intersected, the formation of what was interpreted as a small intersection chamber. Strong winds could prevent any structure from forming, whereas light winds distorted the formation of enclosures resulting in extended linear galleries.

In addition to establishing that complicated structures reminiscent of natural termite mounds can result from very simple rules, these models provide a strong demonstration of the importance of environmental processes in the formation of such mounds. They show that spatio-temporal pheromone properties critically influence construction, and that additional influences such as wind or a net flow of termites can affect building behaviour in interesting ways.

As part of our investigations we reimplemented the Bonabeau et al. (1997) model. Whereas the original model concentrated mostly on characterizing the distribution of active material, we were concerned to understand the entire morphologies of termite constructions - i.e. the active (recently placed) material plus the inactive material that had ceased to exude cement pheromone. Fig. 1 left shows the distribution of active material at the end of a typical simulation. From this figure it is possible to see peaks and troughs in the inactive material which are interpreted by Bonabeau et al. (1997) as pillars. Fig. 1 right shows the total distribution of material (active plus inactive) at the same point in time. It can be seen that the oscillations exhibited by the active material are also present in the total material, but at a fine scale compared to the total amount of material deposited. Rather than appearing as pillars, the overall distribution of building material resembles a high plateau with an irregular surface.

In longer simulations the size of the oscillations remains small in comparison to the height of the plateau 

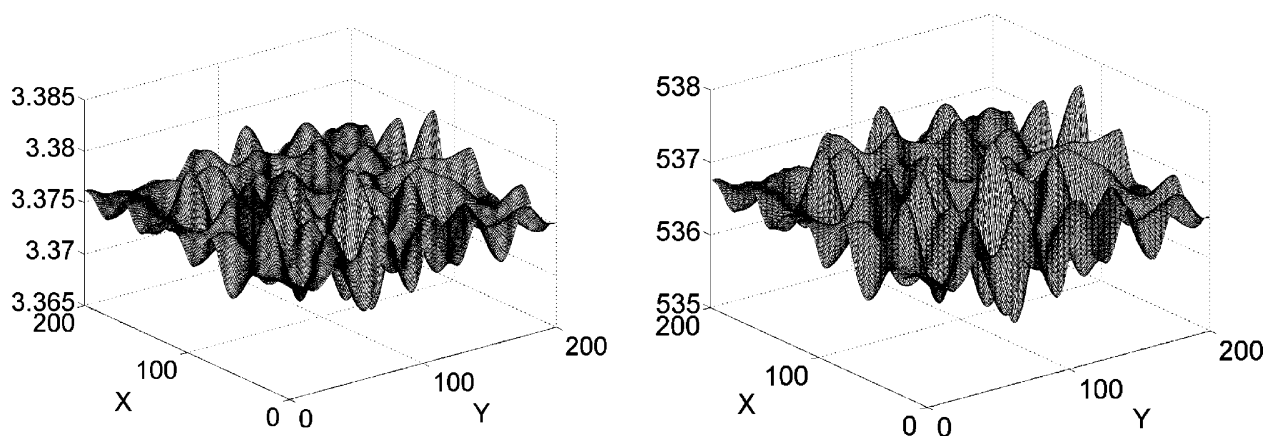

Fig. 1. The formation of pillars from a replication of the Bonabeau et al. (1997) mathematical model. Left-The formation of pillar-like structures of active material. Right-The distribution of inactive material combined with active material (note the difference in vertical scale) demonstrates that the previously observed pillars appear as ripples on the surface of a high plateau.

on which they are appear. We interpret this as meaning that, rather than demonstrating the formation of pillars, the model demonstrates the formation of a state which could result in pillars. Indeed, the model was originally intended to capture only the initial building behaviour of termites rather than some long-term or steady-state outcome (Deneubourg, 1977). After a while, partially built constructions begin to interfere with the movement of termites and pheromones. Since this interference was not captured by the model's set of coupled differential equations it was appreciated that the model is suitable only for capturing the initial symmetry breaking phase of the construction process. It is therefore important that further models of termite construction are able to demonstrate the entire pillar and chamber-formation processes in order to verify the interpretation of earlier results.

Mathematical treatments such as the Deneubourg (1977) and Bonabeau et al. (1997) models are limited in certain important aspects. First, since they do not explicitly represent the third spatial dimension, they cannot directly represent hollow termite-built structures such as arches, domes, and tunnels. Moreover, they neglect any climbing that termite structures might demand. More importantly, as alluded to above, such treatments do not model the logistic constraints on termite movement and pheromone diffusion that are imposed by the physicality of building material.

In both models described above, the presence or absence of built material has no direct effect on either termite movement or pheromone diffusion. This is important, as it prevents the physical consequences of termite construction activity from directly impacting on subsequent termite behaviour, i.e. an important source of stigmergic effects is neglected. For instance, termites may continue to place building material in locations that are physically inaccessible to them, while pheromone may diffuse through solid structures influencing termites that in reality would be ignorant of the pheromone source. Here we label the constraints that these physical realities place on termite behaviour "logistic" since they concern the ability of termites and pheromones to travel from one particular place to another. In systems such as the one considered here, where positive feedbacks operate at many levels, the details of individual agents, their locations, trajectories and past experiences can be significant at the systemic level. By modelling these attributes in the limit or as statistical aggregates, the logistics of who does what to whom, when, and how, can be lost. Individual-based models offer one approach to exploring the effects of these factors. Unfortunately this is often only achieved at the expense of mathematical tractability.

While some models of decentralized insect construction have incorporated logistic constraints of the kind described above, they have not included the role of pheromone diffusion (Bonabeau et al., 1994; Théraulaz and Bonabeau, 1995; Bonabeau et al., 2000) and/or have tackled the problem from an engineering rather than biological viewpoint (Murata et al., 2002; Ladley and Bullock, 2004; Howsman et al., 2004).

In the next section, inspired by a combination of the models mentioned above, we describe a 3-D agent-based model developed in order to explore the impact of logistic constraints on termite construction. It is important to note that while including logistic constraints and a third dimension increases the realism of our model when compared with previous work, we are including these specific factors in order to explore their influence on construction since they might be expected to affect previous results, rather than due to a desire for increased realism, per se. Initial results from this model have been reported briefly elsewhere in the context of engineering and optimization (Ladley and Bullock, 2004). Here, we present biological implications of results from simulations of royal-chamber construction, walkway formation, the effects of wind, and, for the first time, the formation of entrances. We conclude with a discussion of the role of logistic constraints in decentralized termite construction. 


\section{Method}

The simulation was based in a $3-\mathrm{D}$, cubic lattice $(60 \times 60 \times 60)$ with each location containing some combination of diffusing pheromones, simple virtual termites, solid building material, and, sometimes, wind. The model was updated synchronously in fixed discrete time steps. The lattice is initialized as empty of pheromone and building material and open in all directions, save that the lowest horizontal cross-section contains a single layer of inert building material that represents the ground.

\subsection{Pheromone behaviour}

The distribution of each type of pheromone changes over time due to the action of three processes: emission, diffusion, and decay.

Emission: Three kinds of pheromone originate from three different kinds of point source. The queen produces a constant volume of queen pheromone at each time step. Likewise, a trail-following termite produces a constant volume of trail pheromone at each time step. By contrast, the amount of pheromone emitted by each piece of building material is neither constant over time nor unlimited. Rather, each newly placed piece of building material contains an initial finite volume of cement pheromone, $f$, a proportion, $0<r \leqslant 1$, of which is lost to the atmosphere at each time step.

Diffusion: Diffusion between lattice locations that share a common face was modelled as proportional to the pheromone gradient between them using a standard finite volume approach (Hirsch, 1988). If the volume of pheromone at a particular location is $x$ and the volume at one of its diffusion neighbours is $y$ then the change of pheromone may be expressed as $\partial x / \partial t=-\alpha(x-y)$, where $0 \leqslant \alpha \leqslant \frac{1}{6}$ in order to prevent destruction or the creation of pheromone during diffusion. In all results reported here $\alpha=\frac{1}{7}$, which ensures that, at minimum, a pheromone concentration of $\frac{x}{7}$ remains after diffusion, while a maximum of $\frac{x}{7}$ can diffuse to any one diffusion neighbour. Higher values of $\alpha$ would result in more pheromone diffusing to each neighbour than remained in the source whilst lower values of $\alpha$ would lead to slower diffusion with more pheromone remaining at the source location.

Physical constraints on pheromones are modelled by returning any pheromone that diffuses into a location occupied by building material to its source location. The pheromone is then free to diffuse again in the next time step. Note that this scheme ensures that a piece of building material that neighbours other pieces on many sides will emit pheromone at a slower rate than a lone piece of building material as it has less surface area from which to emit. Absorbing boundary conditions assume that locations beyond the 3-D grid are always empty of pheromone.

Decay: Pheromone decay was modelled every time step at a rate proportional to pheromone concentration by multiplying the pheromone level at each location by a decay constant, $0<v<1$.

\subsection{Termites}

The world contains a fixed number of termites, occupied by different tasks (e.g. building, nursing, foraging). Unrealistically, termites do not dynamically change task, but are each fixated on a single behaviour. The number of building termites, $n$, remains constant over the course of a simulation. Each termite can sense the levels of pheromones at its current location and the pheromone gradients in each direction.

Movement: Termites may move to any adjacent location on the cubic lattice, however, the choice of movement is physically constrained. Of the 26 possible adjacent locations available to a termite, it may only move to one that is unoccupied by building material (including the "floor"). Additionally, termites may only move to locations that neighbour locations occupied by building material. The first constraint prevents termites moving through walls, while the second constraint forces termites to move across surfaces, preventing them from flying around the world. Furthermore, certain locations that satisfy these two constraints are nevertheless deemed inaccessible in the following sense.

In the cubic lattice that we employ, a location that is empty of building material and shares exactly one edge with a termite's current location is inaccessible if the other two locations that share the same edge both contain building material. Likewise, a location that is empty of building material and shares exactly one point (vertex) with a termite's current location is inaccessible if three of the locations that share the same point contain building material and do not share a common face (see

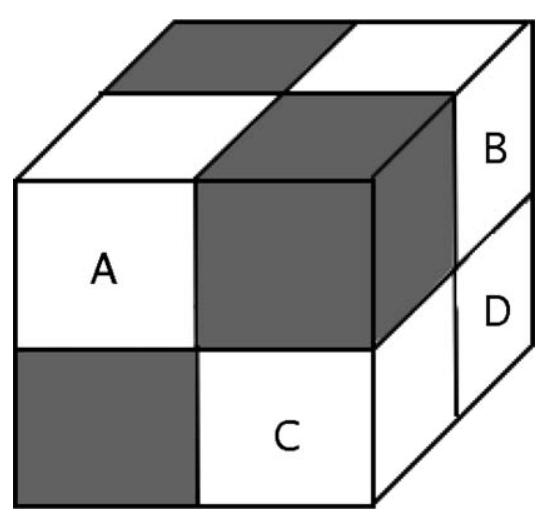

Fig. 2. Empty locations B, C, D, and E (occluded) are inaccessible from location $\mathrm{A}$ (and vice versa). 
Fig. 2 for clarification). These constraints are intended to prevent termites moving through walls.

If a termite leaves the lattice, which it may do freely, or cannot move to any adjacent location, it is discarded and a new replacement termite is introduced to the lattice.

Work carried out by Bruinsma (1979) has demonstrated that the movement of termites engaged in building activity is biased by the presence of cement pheromone. It was shown (i) that termites tend to climb a cement pheromone gradient and (ii) that the chance of a termite following a pheromone gradient is proportional to its strength. In order to capture this behaviour in the model, the movement of builder termites is implemented as follows. A new location is chosen from the legal alternatives using a roulette-wheel constructed to reflect relative cement pheromone gradient strengths. However, with probability inversely proportional to the gradient strength of the selected direction, a random legal move is made instead. The roulette wheel ensures that model termites tend to travel up pheromone gradients, whilst the possibility of a random move ensures that, on average, weak gradients exert less influence on termites than strong gradients.

A termite moves $m$ times in this fashion every time step. Since termites move relatively fast by comparison with pheromone diffusion, for all results reported here, $m=5$. Termites are not prevented from entering locations already occupied by other termites. In reality termites are much larger than the pieces of material that they deposit, however, in order to make our model tractable it was necessary to represent each termite as occupying a single lattice location. This location can be considered to be the head of the termite, i.e. the place it would perform its building and sensing activities. It seems plausible that two termites could be collecting sensory information from the same location at the same time, although a more realistic model would include physical interference between termites and prevent termites from moving through gaps that are unrealistically small.

Block placement: In order for a piece of building material to be placed at a particular location, the level of queen/trail pheromone at the site must lie within a predefined range $([0.1,0.5]$ for all results presented here). Additionally the site must meet at least one of the following three conditions, which are intended to impose a crude physics on the world:

1. Either the location immediately underneath or immediately above the site must contain material.

2. The site must share a face with a horizontally adjacent location that contains material and satisfies (1).

3. One face of the site must neighbour three horizontally adjacent locations that each contain material.
The first constraint allows vertical stacks to be built. The second allows these stacks to be extended horizontally to a limited degree, while the third allows the gradual construction of elevated horizontal surfaces if sufficient support is present.

If these conditions are met, a block will be placed at the termite's current location with fixed probability, $p$, the termite will be removed from the lattice, and a replacement introduced. This is intended to represent the constraint that termites must forage for building material. We do not explicitly model this activity, since it would require some representation of sources of building material and a more sophisticated locomotion strategy on the part of the simple termites. Notice that once placed, building material cannot be removed or relocated.

Trail-following: In addition to the queen, and buildertermites, a third class was modelled. These termites lay and follow trail pheromone, but are not involved in any building activity. (Currently, the model does not allow for trail-following termites or building-termites to change role during the simulation.) They represent termites who are leaving the nest to forage for food or returning to the nest having foraged. A group of adjacent, ground-level entry points are specified at the edge of the lattice. At each time step, and with fixed probability, $c$, each of a fixed number of termites may enter the lattice at a randomly chosen entry point. Each trail termite moves across the lattice in a direction roughly perpendicular to the lattice edge at which they enter. However, trail termites are not restricted to move in a straight line. In order to impose a kind of "directed drunkards walk" on the termite trajectories, we adapted an approach employed by Deneubourg in the 2-D case (Deneubourg et al., 1989). A trail termite is allowed to move to any down-stream location that shares a face, edge or corner with its current location and is physically accessible. Trail termites are attracted to trail pheromone in the same way that builder termites are attracted to cement pheromone. If a termite moves off the lattice it is removed.

Nursing: The final termite behaviour considered is intended to represent the actions of those individuals that are concerned with tending to the queen termite, either by bringing food, or removing larvae. These "nursing" termites closely resemble the path-following termites described above. Each of a set number, $e$, of nursing-termites start the simulation at a random location constrained to lie a specified distance, $d$, from the queen-termite in a random direction. Nursingtermites move in a similar manner to the trail-following termites, with the exception that they are constrained to move towards the queen instead of simply across the world. When the queen is reached the movement behaviour reverses; the nursing-termites are forced to move away from the queen (in any direction). This 
behaviour continues until the termite reaches a distance, $d$, from the queen's location, at which point it again reverses direction and moves as originally specified. Like trail-termites, at each time step a unit of trail pheromone is deposited. In addition to being attracted towards trail pheromone there is a small fixed probability, $b$, that a nursing-termite will make a random movement ignoring pheromone gradients. This random movement is necessary in order to help prevent termites becoming trapped in locations from which they are unable to escape, e.g. locations from which there are no possible moves towards/away from the queen.

\subsection{Wind}

Wind was modelled in a deliberately simple manner, originating at a constant fixed strength, $0<s<1$, from one vertical face of the lattice, travelling perpendicular to this face, horizontally across the world, flowing around built structures, and influencing pheromone diffusion. In general, therefore, wind flows from one upwind vertical cross-section of lattice, $a_{i}$, to the adjacent, down-wind, vertical cross-section, $a_{i+1}$. Wind strength in $a_{i+1}$ can be calculated in the following manner. For every location, $u$ in $a_{i}$, calculate the number of locations in $a_{i+1}$ that share an edge, corner or face with $u$ and are empty of building material. Divide the strength of wind at $u$ equally among these locations in $a_{i+1}$, or (unrealistically) discard the value should no downstream locations be empty of building material. All boundary conditions were reflective with the exception of the down wind boundary through which wind could pass freely.

In areas lacking building material, this approach will result in wind strength remaining constant, as the strength at each location will be distributed evenly across down-wind locations. In areas with obstructions, wind strength will increase near exposed surfaces, but be reduced in "sheltered" areas, as the wind flows around the obstruction.

At each location, wind transfers a proportion of pheromone equal to its strength, $s$, to the location immediately down-wind (unless that location is occupied by building material). While wind can thus only carry pheromone in one direction, in combination with diffusion, it is capable of transporting pheromone around structures effectively.

\section{Results}

Simulations were carried out to examine a range of scenarios, including those considered in previous models and novel situations intended to highlight the role of logistic constraints. This allowed us first to verify that the results of Deneubourg (1977) and Bonabeau et al.
(1997) extended to 3-D, and then to explore the extent to which they were affected by imposing a degree of physicality on the interactions between built structures, termites and pheromones. First, pillar formation, wall building and dome completion were explored in the context of a pheromone gradient established by a stationary queen. The influence of wind on this process was examined. Then the tendency for a single flow of trail-termites to encourage tunnel building, and for a pair of orthogonal flows to bring about a tunnel intersection were investigated. Finally, we tested a mechanism for introducing and maintaining an entrance in a royal chamber that is entirely dependant on the physical constraints imposed on pheromones and termites by built structures. This mechanism involves adding trail-laying nursing-termites to the initial royal chamber scenario and relies upon interactions between trail-, cement- and queen-pheromone-driven behaviours. The results of these investigations are presented below and their implications discussed in the subsequent Discussion section.

\subsection{Royal chambers}

The queen, represented by one or more contiguous blocks in the form of a rough half-cylinder, is placed at the centre of the world in contact with the ground. Each block is a fixed-rate source of queen pheromone. Throughout the simulation the queen remains stationary and is somewhat equivalent to building material in that she obstructs the flow of pheromone, and impedes movement of termites, allowing them to climb over her though not to build on her.

The simulation is initially run for 1000 time steps without the presence of builder termites to allow the queen's pheromone template to become established. At this point termites are randomly allocated around the outside of the world in contact with the ground, and the model proceeds as described above. Unless otherwise noted the simulations were run with 300 building termites $(n)$, a probability of block placement $(p)$ of 0.1 , a pheromone evaporation rate $(v)$ of 0.1 and a pheromone output rate $(r)$ of 0.5 .

Figs. 3 and 4 depict the construction of a royal chamber without wind, and under mildly windy conditions, respectively. First, pillar-like structures are formed at roughly regular spatial intervals, and at a specific distance from the queen. Subsequently, these pillars merge to form a wall that encircles the queen. Finally, the termites achieve a complete dome encompassing the pheromone source. In the absence of any disturbance, termites achieve a roughly hemispherical structure centred on the queen, that may echo the queen's physical shape if she is large enough in relation to the dome (see Fig. 3). However, in Fig. 4 the shape of the chamber has been influenced by wind blowing from 


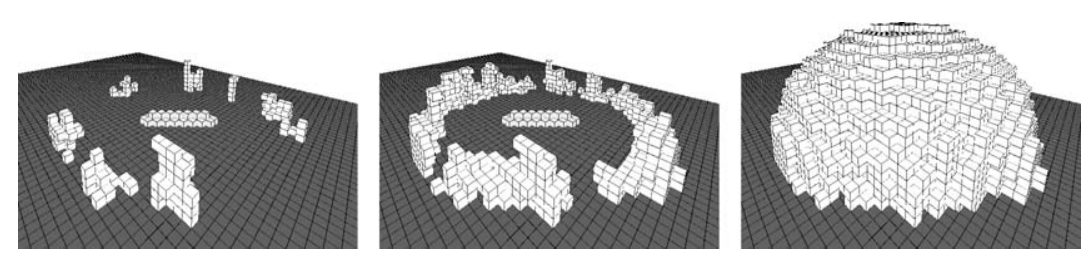

Fig. 3. A royal chamber being constructed. Parameters: $f=400, r=0.5, \alpha=\frac{1}{7}, v=0.1, p=0.1, n=300, m=5, s=0.0$.
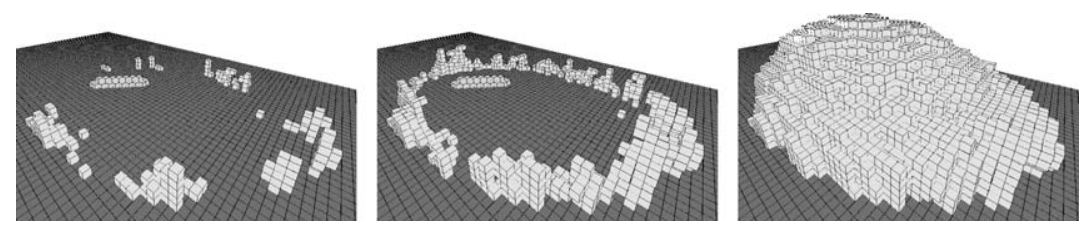

Fig. 4. A royal chamber being constructed under mildly windy conditions (wind emanates from the upper-left lattice edge). Parameters as Fig. 2, except: $s=0.15$.

the upper-left face of the lattice. As a result, the chamber is not centred on the queen, and is distorted in both its horizontal and vertical profile, e.g. exhibiting a steeply rising exposed face and a more gradually descending, sheltered, down-stream slope. At higher strengths, wind can prevent a chamber from being completed successfully, or even prevent any construction from becoming established at all.

\subsection{Recruitment}

The number of building termites used in the construction of the royal chamber was varied while all other parameters were held constant. After 200 building steps the amount of material placed by the termites was recorded. Fig. 5 shows the results collected, averaged over ten runs. With low numbers of termites the amount of work done per termite is relatively small. However, as the number of termites is increased, the amount of building work per termite increases at an increasing rate. With very high numbers of termites, work rate saturates. By contrast with models neglecting logistic constraints, these results are qualitatively similar to those observed in real termite colonies (Bruinsma, 1979).

\subsection{Covered walkway}

A narrow flow of termites across the world is introduced by allowing trail termites to enter at ground level from central locations along one lattice edge. Builder termites enter the lattice from random edge locations after 1000 time steps. Fig. 6 shows the resulting tunnel, partially formed, and an internal view. Construction of the tunnel walls obviously precedes completion of the roof. At the end of the simulation the tunnel is straight, quite regular in cross-section, and clear of obstructions. Notice that, unlike a previous model (Bonabeau et al., 1997), the trail and distribution

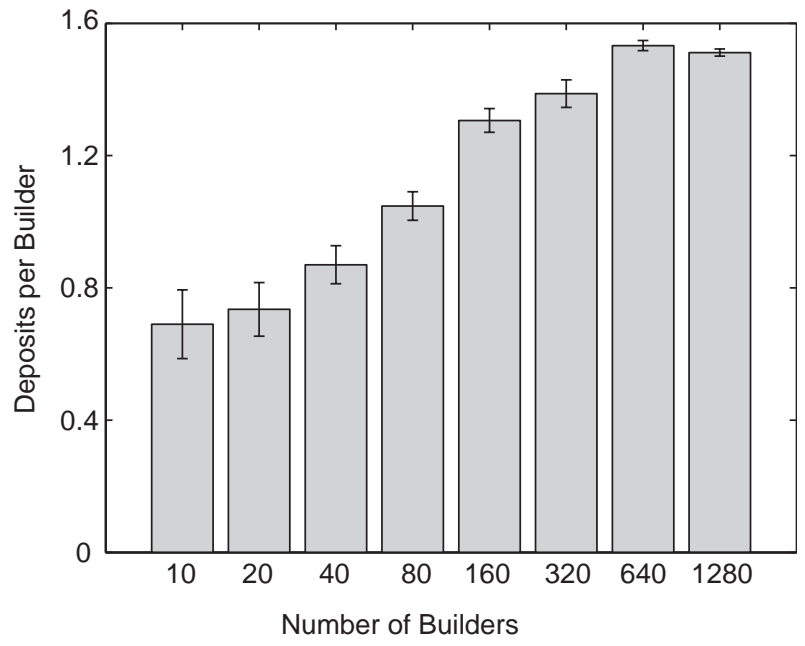

Fig. 5. A graph showing the amount of work done per termite versus the number of termites present in the simulation. Each error bar represents the standard error from the results of 10 replicates. Note the geometric scale on the abscissa. The sinusoidal shape is similar to that observed in real termites (Bruinsma, 1979).

of trail pheromone are not explicitly defined, but arise from the ongoing activity of trail termites.

In many cases the final tunnel formed exhibits a high degree of flaring at the far end. Fig. 7 shows the process that causes this. In the first image a high-concentration band of trail-pheromone runs across the world. The next four images depict the formation of a secondary side trail. This is caused by the presence of building material along the sides of the main trail. By obstructing trailtermite movement, this material deflects termites that encounter it and encourages them to deviate from the central trail. As tunnel walls begin to be constructed along these side trails, more deflection of trail termites occurs increasing the down-stream "flaring" of the tunnel. The final image shows the completed flared tunnel. 

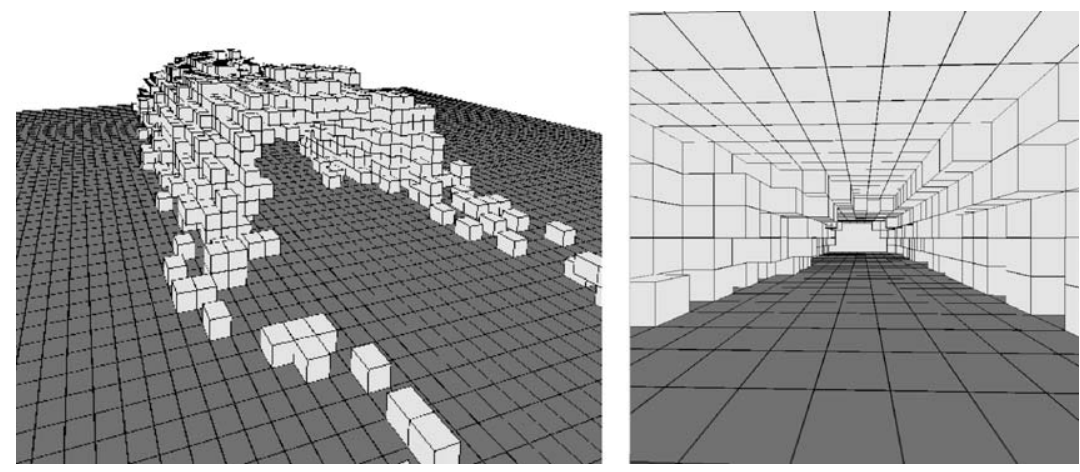

Fig. 6. A covered walkway is constructed. Parameters as Fig. 2, save that a flow of trail termites has been introduced: $t=10, c=0.5$. At each time step, between zero and 10 builder termites enter the lattice, with probability 0.5 per termite. The tunnel's interior is clear of obstructions, and the cross-section is quite regular.
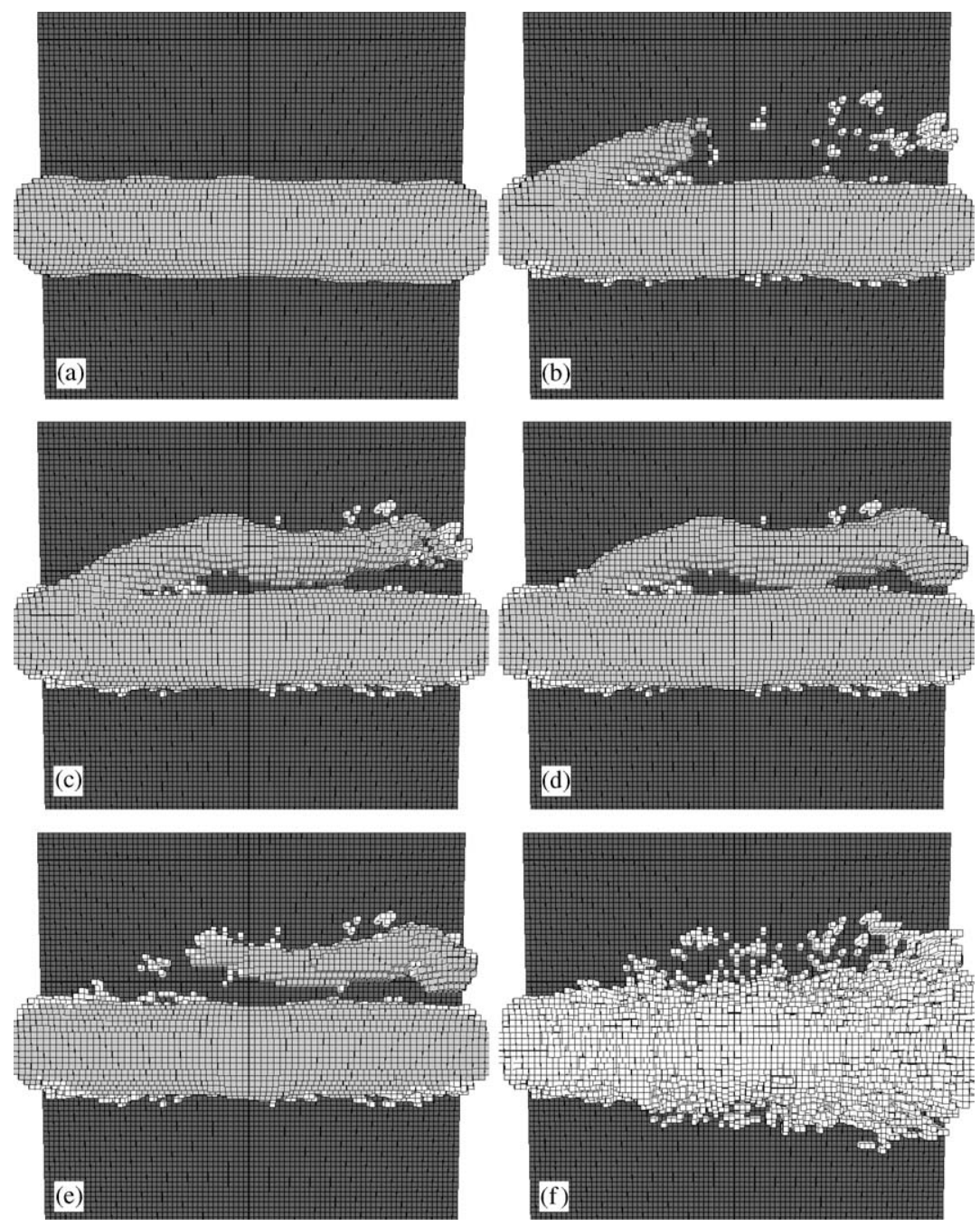

Fig. 7. Stages in the construction of a covered walkway, viewed from above (floor in heavy tone; trail pheromone in medium tone; building material in light tone). The tunnel formed is flared by deflected trail termites. See text for explanation.

This flaring is eliminated when building termites are constrained to enter from the same locations as the trail termites. Fig. 8 demonstrates the formation of a tunnel under this constraint. Building termites that enter from the same location as the trail termites are "guided" by the partially-built structure such that the tunnel does not flare. As first the walls, and then the roof are constructed, they funnel the building termites to the 

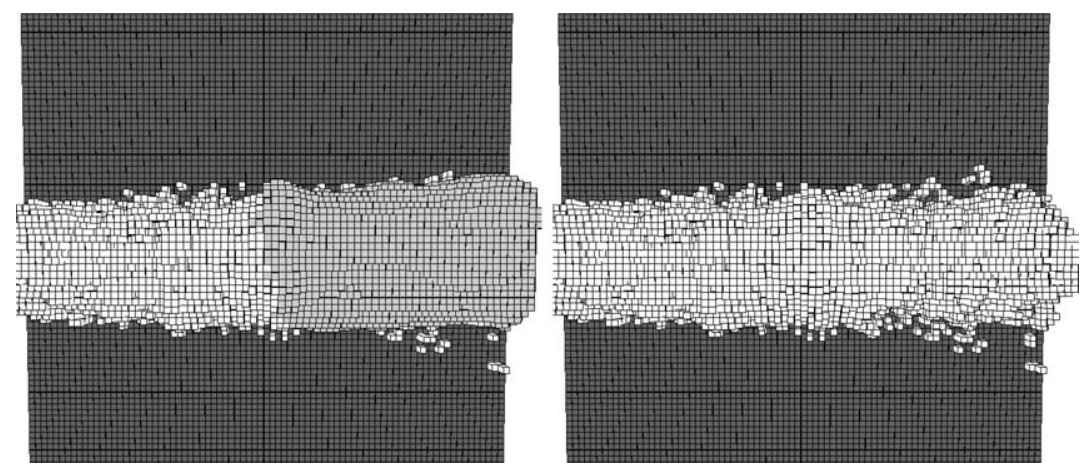

Fig. 8. The construction of a non-flared covered walkway viewed from above. Parameters as previous figure. Builders now enter from the same location as trail termites. Trail pheromone is again depicted in medium tone.
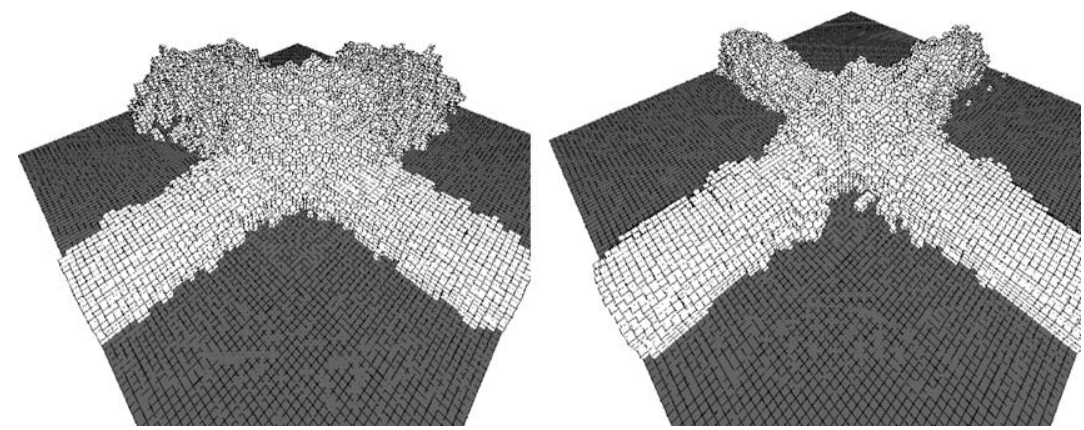

Fig. 9. Two examples of cross-road formation. Parameters again as Fig. 2, save that two perpendicular streams of trail termites are introduced: $t_{1}=10, t_{2}=10, c=0.5$. At each time step, between zero and 10 trail-termites enter the lattice, with probability 0.5 per termite. Left-Builder termites enter from random locations along any edge of the ground plane. Some "flaring" of the down-stream sections of tunnel tends to occur. RightBuilders enter from the same entry points employed by trail termites. Flaring is suppressed.

location at which building work must occur next. Consequently there are fewer pieces of stray material that might deflect trail termites away from the trail. In addition by steering the building termites to the leading edge of the building area there are fewer building termites available to place material near any side trails which do form.

\subsection{Tunnel intersections}

Two perpendicular flows of termites are introduced, crossing at the centre of the ground plane. Fig. 9 depicts the cross-road structures that arise. Despite the potential for interference between trail pheromones from each trail, and between the building work at each tunnel (especially given the stochastic rate at which both trails and tunnels are established), each tunnel remains unobstructed internally, and a working covered crossroad junction is achieved.

Fig. 9 left depicts a scenario in which builder termites always enter the world from a random location along the ground-level edge of the lattice. The covered walkways that result reliably exhibit a tendency to "mushroom" at their down-stream ends. Constraining the building termites to enter at the same locations employed by the trail termites extinguishes this tendency (Fig. 9 right) for the reasons described above. In both cases, however, the interior of the tunnel remains clear of obstructions.

\subsection{Chamber entrances}

Simulations of the construction of the royal chamber were run as described in Section 3.1. The simulation was modified to include a number of nursing-termites equal to the number of building termites (300). In addition, the queen was modified to be a point source to avoid biasing the location of any entrances that might form. Fig. 10(a) shows the state of the world after 50 time steps. The initially randomly distributed nursing-termites have self-organized into six trails from the queen to the edge of the world due to positive feedback from the pheromone that they have laid. Fig. 10(b) shows the same simulation 450 time steps later. Competition between trails coupled with the tendency for builder termites to obstruct trails that are less frequently used 

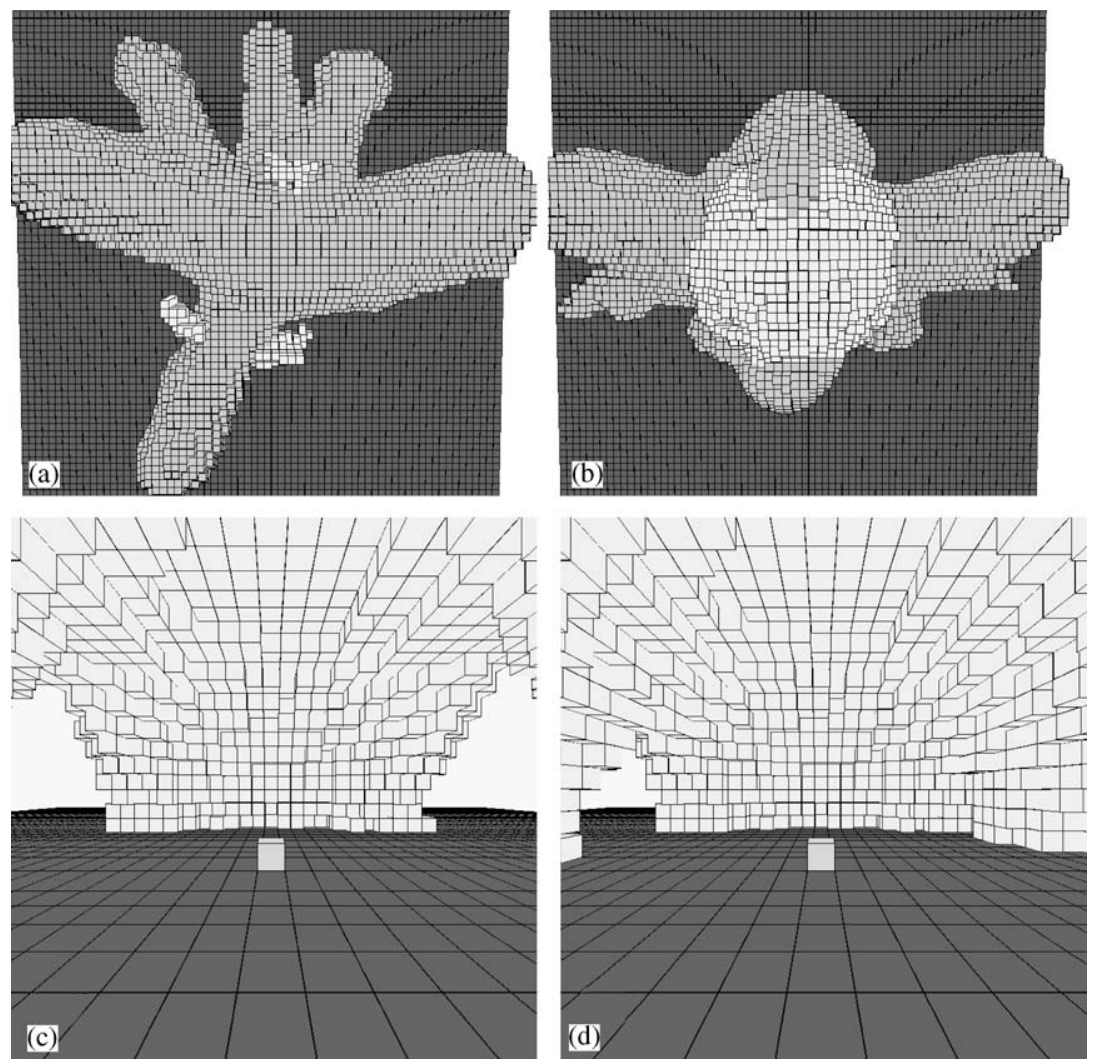

Fig. 10. An example of entrance formation. Parameters as Fig. 2, except $e=300$ (300 nurse termites are added) (a) 50 time steps: several (medium tone) pheromone trails between a central queen and the lattice periphery have formed (shown from above). (b) 500 time steps: only two trails remain. (c) 500 time steps: A view from inside the dome. (d) 800 time steps: only one entrance remains.

has resulted in four of the trails being abandoned. Fig. 10 (c) shows the same situation from a viewpoint inside the dome. After 200 further time steps, only one of the entrances remains (Fig. 10(d)).

Given that sufficient numbers of nursing-termites continue to follow a trail running through a chamber entrance, the entrance will remain unobstructed indefinitely. As described above, nursing termites have a slight tendency to move at random in order to help avoid situations in which they become trapped by built structures that prevent them moving directly toward/ away from the queen. Despite this tendency, nursing termites do occasionally spend significant amounts of time struggling to circumvent obstructions. In some cases, the number of "trapped" nursing termites can grow, reducing traffic in and out of the chamber entrances. This can significantly weaken the pheromone trails leading in and out of the chamber, allowing building termites to succeed in blocking all chamber entrances.

Although this method of entrance formation is capable of maintaining a single chamber exit, royal chambers found in natural termite colonies frequently have multiple entrances (Bonabeau et al., 1997). However, with a slight modification the model also exhibits this behaviour, as shown in Fig. 11. If nursing termites have specific destinations on the periphery of the lattice, as would be expected in real termite colonies, there is the potential for multiple entrances to form.

Instead of attempting to merely move away from the queen in any direction, each nursing termite is given a specific destination on the periphery of the lattice. Fig. 11 demonstrates the case of two specific locations on the edge of the world. If these destinations are separated by a sufficiently large angle, multiple entrances will form (Fig. 11).

\section{Discussion}

The primary result exhibited by the model is that, pleasingly, the introduction of physical limitations on pheromone diffusion and termite movement have not prevented the construction of structures suggested by previous models that neglected such logistic constraints (Deneubourg, 1977; Bonabeau et al., 1997). In addition to this verification of previous results, we have shown how this physicality can be exploited by termites in the construction of more efficient or sophisticated structures. 


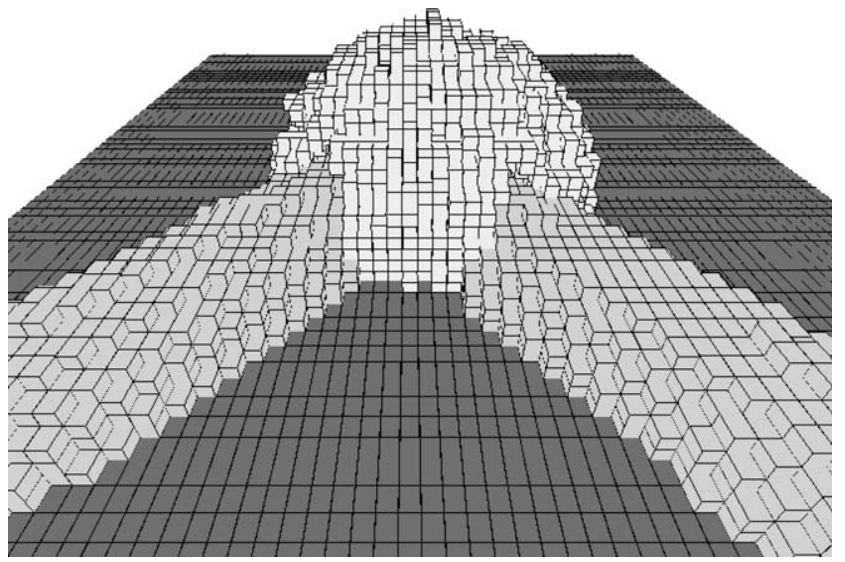

Fig. 11. An example of the formation of multiple entrances. Parameters as previous figure, except $e=300$. The added nursing termites move towards specific locations on the periphery of the world as evidenced by (medium tone) pheromone trails.

As reported by Deneubourg (1977), pillar formation can be driven by simple positive feedback involving a cement pheromone. His results from 1-D and 2-D models built on differential equations can be supported by our 3-D agent-based model. For instance, in our simulations, pillars form at regular spatial intervals in the early stages of the construction of the royal chamber (see Fig. 3). Moreover, whereas the formal models presented first by Deneubourg (1977) and extended by Bonabeau et al. (1997) failed to exhibit the sigmoidal work-rate amplification curve (see Fig. 5) characteristic of the stigmergic positive feedback that drives natural termite recruitment, the current model did exhibit a qualitatively equivalent relationship.

Our model also agrees with predictions of Bonabeau et al. (1997) that the introduction of a source of diffusing queen pheromone can first guide the construction of pillars and subsequently an encircling wall. Our results show that this process can result in a fullyformed closed royal chamber. The manner in which our results are influenced by wind also agrees with previous findings, which reported a distortion of the horizontal cross-section of the royal chamber. However, our treatment of the third dimension also allows us to explore distortion to the elevation of the chamber.

Finally, the Bonabeau model suggested that an artificially imposed flow of termites emitting trail pheromone could result in the construction of walkways. Our simulations extend these results by showing that these structures tend to become covered walkways over time, and remain clear of building material even when built structures have the potential to interfere with trail laying termites (see Fig. 6). Previous speculation by Bonabeau et al. (1997) that two intersecting trails could give rise to the formation of a cross-road structure are confirmed by our model, which additionally demon- strates that such an intersection will not tend to become blocked by building activity (see Fig. 9).

In addition to confirming work from previous models, our approach has generated new insights. First, and most generally, it is perhaps remarkable that the introduction of physical constraints on termite motion and pheromone behaviour did not more greatly disrupt the structures achieved. It was possible, for instance, that the simple agents that we modelled could have found it difficult to complete a closed structure such as a dome, since those termites inside the dome might have experienced a largely homogeneous pheromone distribution, while those outside may have been confused by any pheromone that spilled from the holes remaining to be filled. Similarly, one might have imagined that as a tunnel was formed and enclosed the trail within it, it would become difficult for trail pheromone to escape, distorting the pheromone distribution in such a way as to interfere catastrophically with new building activity. Moreover, in the current model, when a piece of building material is placed it cannot be removed, meaning that, for instance, should tunnel blockages arise, they cannot be rectified, making tunnel completion in such situations impossible. It is of course true that natural termites do pick up previously deposited building material, however, the previous models that we are extending do not include this behaviour. The impact of this additional activity thus remains an open question. However, it is interesting to note that our results demonstrate that "picking up" behaviour is not required in order to generate well-defined, obstacle-free termite architectures. This is especially significant, since results from our replication of a previous model (Bonabeau et al., 1997) reported in Section 1.2 suggest that unwanted inactive material might build up across the surface being built upon, interfering with the construction of tunnels and chambers.

In order to implement constrained movement and diffusion we introduced an explicit third dimension. This alone could have introduced dramatic differences between the behaviour observed in our system and in previous models. Climbing, for instance, plays no part in 2-D models, but is important in the current work, since agents must be able to reach a location in order to build there. Despite this, all of the structures suggested by the two relevant previous models were achieved. However, the fact that, in our model, it is harder to reach some locations than others causes agents to build more slowly in hard to reach places like roofs of structures, or the tops of pillars, which require time consuming ascents compared to the walls which are at ground level.

Predictably, as the strength of simulated wind increased the building process became slower until a point was reached where no building was possible. While the effect of wind may simply be to disturb a pheromone template to the extent that builders are not 
encouraged to lay down building material at any location, it also has a more subtle influence on the ability of newly-formed structures to recruit builder termites. Without wind, cement pheromone is free to diffuse in every unobstructed direction, attracting termites to the emitting structure from a wide area. In the presence of wind, however, the cement pheromone cannot diffuse as effectively, hampering the recruitment of up-wind builder termites. This ensures that mildly windy conditions result in differential construction activity.

Rather than being severely limited by the physical obstruction imposed by the results of their own building behaviour, it appears that in some cases these effects can increase the efficiency of the building work and prevent some "problematic" kinds of construction activity such as the tendency for tunnels to "flaring" observed in Fig. 9. This "overbuilding" stems from a combination of two factors, (i) a slight "fanning" of the trail laid by the termites due to their stochasticity and amplified by confusion at the intersection of two perpendicular pheromone trails, and (ii) a tendency for builder termites to approach the trail from all sides. These conditions lead to building behaviour near the outer edge of the trail "fan", which is amplified and ultimately results in wide tunnel walls. By contrast, where builder termites enter the lattice at the same points as the trail laying termites, they tend to begin building at a regular distance from the trail mid-line. As a tunnel is formed in this way, it limits the movement of builders, directing them to the tunnel end and preventing them from reaching the outer edge of the "fanned" distribution of trail pheromone. In this way, despite the inability of the trail termites to form a narrow, fixed-width pheromone template, building work is able to produce a narrow, fixed-width tunnel.

Finally, a novel contribution made by the model is to demonstrate the manner in which multiple pheromone processes can interact to maintain an entrance in a royal chamber both as it is built and subsequently. As the trails laid by nursing-termites compete with each other for followers, and the builder termites extinguish any trails that are unused by obstructing them with building material, the termites come to increasingly use a single entrance and in doing so prevent it from being blocked by building work. This mechanism relies critically on the ability of the material being laid to obstruct both the movement of termites and pheromones and thus would not have been discovered in a model neglecting to include these factors.

\subsection{Future work}

Currently, several aspects of the model are underdeveloped, or unexplored. Both the implementation of wind, which currently cannot support swirling eddies or any kind of back-flow, and the crude physics of construction, could be improved. The latter might benefit from an approach similar to that taken by Funes and Pollack (1997) in their work on simulating Lego structures. Currently, it is still possible for some unrealistic or impossible structures to be built. A more sophisticated physics might encourage termites to adopt more complicated strategies in order to achieve largescale structures.

As noted above, some logistic factors have not been incorporated into our model. Currently, termites do not obstruct one another's movement, and, unrealistically, are assumed to occupy roughly the same amount of space as a piece of building material. In addition, for reasons of simplicity, we do not yet explicitly model the tasks involved in discovering raw materials or transporting them to the construction site. The significance of these factors remains to be seen.

In general, the behaviour of the artificial termites has a great deal of scope for improvement. In particular the movement rules for the nursing-termites are very simple, and can lead to the termites becoming trapped on the outside or inside of the dome. An improved movement algorithm would prevent this by allowing termites to pursue alternative routes when their path was blocked.

There is, however, a more fundamental problem with the behaviour of the termites. Due to the lack of an explicit model of job allocation, several different types of termite are modelled, i.e. building, trail-following, nursing, etc. However, in real termite colonies it is unlikely that these fixed distinctions exist. Instead termites change roles freely depending on environmental stimuli. An important addition to the model would be to allow the termites to change roles during the simulation in a similar fashion, e.g. from building to nursing. In doing so, the model could be used to explore the mechanisms underlying role switching in termites. It remains to be seen, however, how such dynamic role swapping could be implemented.

More generally, closer comparison between the current model and the detailed behaviour of previous mathematical models is required, and a more extensive characterization of the simulation's dependencies on parameter values and initial conditions should be undertaken.

\section{Conclusion}

In conclusion, we have shown that physical and logistic constraints imposed on termite construction by the results of their own building behaviour do not prevent the formation of several structures reminiscent of natural termite architecture. Indeed, it appears that in some cases these constraints are important or even 
necessary in order for termites to achieve efficient, effective constructions.

\section{Appendix A. Technical}

Here we include some additional detail of the algorithm used to simulate termite construction. Where possible, this is represented in pseudocode, and is anotated where appropriate. Our implementations of diffusion and wind are not described further here as they are detailed in Sections 2.1 and 2.3, respectively. For parameter values, please consult the body of the paper (default or representative values are given in parentheses).

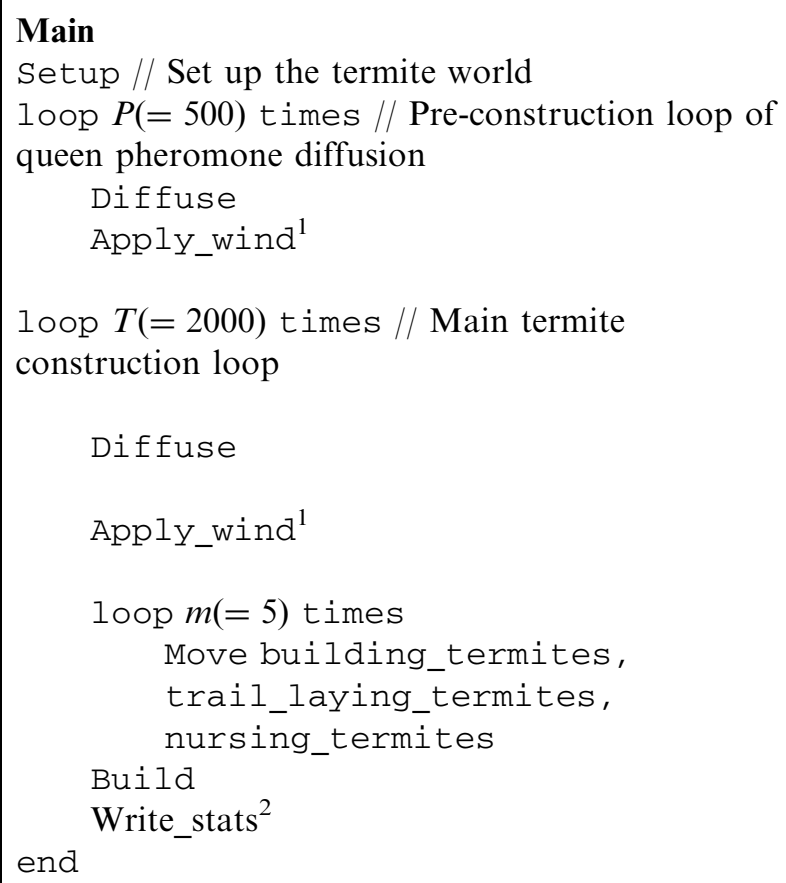

${ }^{1}$ This function is only called for scenarios in which wind is being simulated.

${ }^{2}$ This function may be called less often in order to economize on disk space.

\section{A.1. Setup}

The termite world is represented as a number of 3-D matrices with dimensions $x(=60), y(=60)$, and $z(=60)$ - one matrix for each pheromone distribution and one for representing the location of building material. A list of $N(=100)$ building termites holds the location of each building agent. Similar lists held the locations of trail laying termites and nursing termites where they were involved.

Before the algorithm enters the main loop, the ground is added, and, where required, a queen termite is added. Building termites, and, where needed, trail-laying termites and nursing termites are also given initial locations at the edge of the matrix, adjacent to the ground.

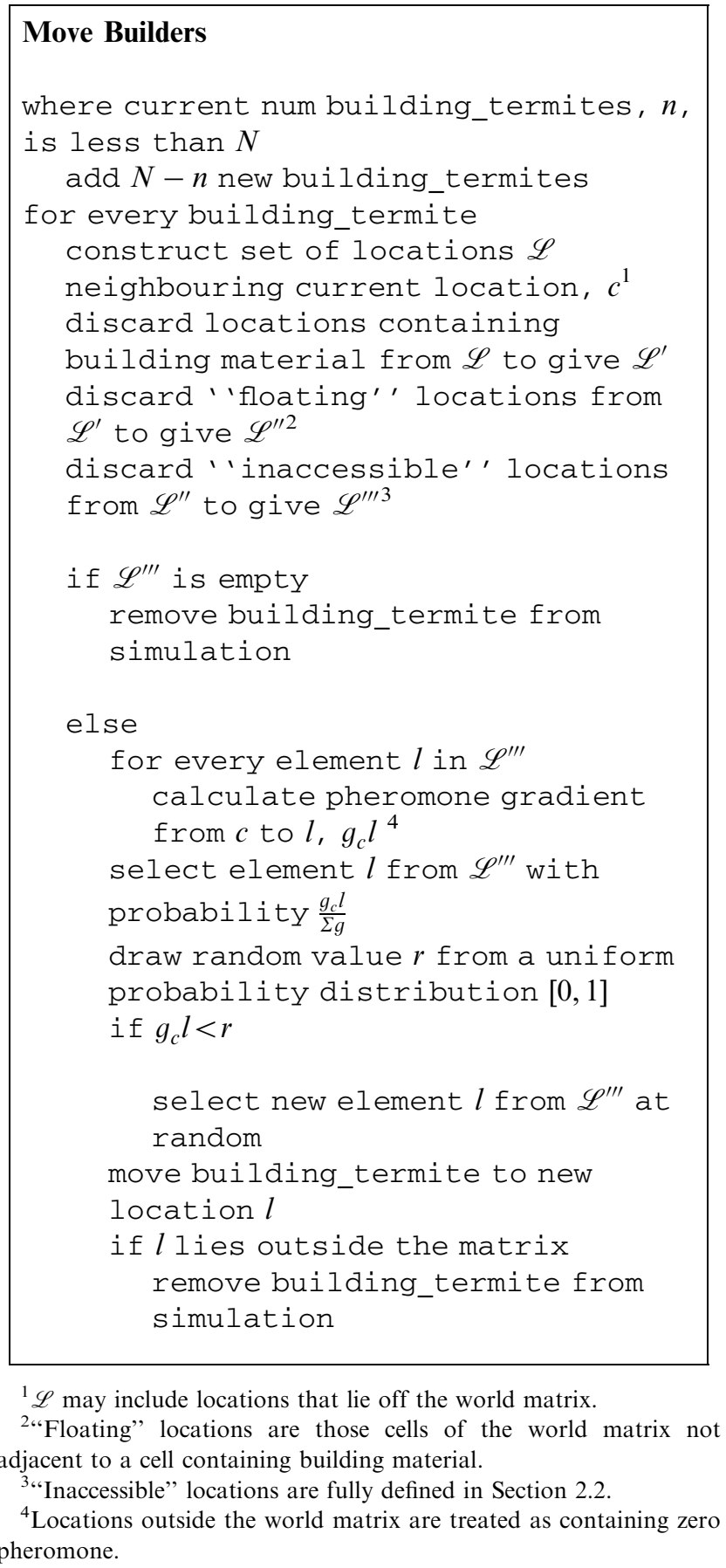

\section{A.2. Move_trail_laying_termites}

As above, save that locations that are not "downstream" of the current location c are discarded from $\mathscr{L}^{\prime \prime \prime}$ to give $\mathscr{L}^{\prime \prime \prime \prime}$.

An element is selected from $\mathscr{L}^{\prime \prime \prime \prime}$ with probability proportional to the associated trail pheromone gradient. 
The algorithm then proceeds as described above for building termites.

\section{A.3. Move_nursing_termites}

As described above for trail-laying termites save that, when approaching the queen termite, a further subset $\mathscr{L}^{\prime \prime \prime \prime}$ is constructed by discarding locations from $\mathscr{L}^{\prime \prime \prime}$ that do not lie in the direction of the queen. When moving away from the queen, $\mathscr{L}^{\prime \prime \prime \prime}$ is constructed by discarding those locations that $d o$ lie in the direction of the queen.

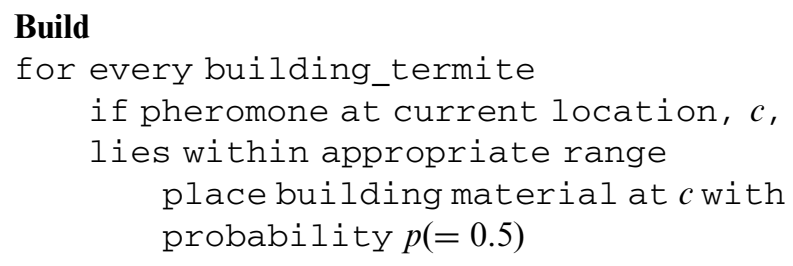

\section{References}

Bonabeau, E.W., Théraulaz, G., Arpin, E., Sardet, E., 1994. The building behavior of lattice swarms. In: Brooks, R.A., Maes, P. (Eds.), Fourth International Conference on Artificial Life. MIT, Cambridge, MA, pp. 307-312.

Bonabeau, E.W., Théraulaz, G., Deneubourg, J.-L., Franks, N.R., Rafelsberger, O., Joly, J.-L., Blanco, S., 1997. A model for the emergence of pillars, walls and royal chambers in termite nests. Philos. Trans. Roy. Soc. Lond. Ser. B 353, 1561-1576.

Bonabeau, E.W., Guérin, S., Snyers, D., Kuntz, P., Théraulaz, G., 2000. Three-dimensional architectures grown by simple 'stigmergic' agents. BioSystems 56, 13-32.
Bruinsma, O.H., 1979. An analysis of building behaviour of the termite Macrotermes subhyalinus (Rampbur). Ph.D. Thesis, Landbouwhogeschool, Wageningen, Netherlands.

Courtois, P.-J., Heymans, F., 1991. A simulation of the construction process of a termite nest. J. Theor. Biol. 153 (4), 469-475.

Deneubourg, J.-L., 1977. Application de l'ordre par fluctuations la description de certaines tapes de la construction du nid chez les termites. Insectes Sociaux 24, 117-130.

Deneubourg, J.-L., Goss, S., Franks, N., Pasteels, J.-M., 1989. The blind leading the blind: modelling chemically mediated army ant raid patterns. J. Insect Behav. 2, 719-725.

Deneubourg, J.-L., Théraulaz, G., Beckers, R., 1992. Swarm-made architectures. In: Varela, F.J., Bourgine, P. (Eds.), First European Conference on Artificial Life. MIT, Cambridge, MA, pp. 123-133.

Funes, P., Pollack, J., 1997. Computer evolution of buildable objects. In: Husbands, P., Harvey, I. (Eds.), Fourth European Conference on Artificial Life. MIT, Cambridge, MA, pp. 358-367.

Grassé, P.-P., 1984. Termitologia Tome II-Foundations des societés - construction. Masson, Paris.

Hirsch, C., 1988. Numerical Computation of Internal and External Flows, vol. 1: Fundamentals of Numerical Discretization. Wiley, Chichester.

Howsman, T.G., O'Neil, D., Craft, M.A., 2004. A stigmergic cooperative multi-robot control architecture. In: Pollock, J., Bedau, M., Husbands, P., Ikegami, T., Watson, R.A. (Eds.), Ninth International Conference on Artificial Life. MIT Press, Cambridge, MA, pp. 88-93.

Ladley, D., Bullock, S., 2004. Logistic constraints on 3d termite construction. In: Dorigo, M., Birattari, M., Blum, C.M., Gambardella, L., Mondada, F., Stutzle, T. (Eds.), Fourth International Workshop on Ant Colony Optimisation and Swarm Intelligence. Springer Lecture Notes in Computer Science. Springer, Berlin, pp. 178-189.

Murata, S., Yoshida, E., Kamimura, A., Kurokawa, H., Tomita, K., Kokaji, S., 2002. M-tran: self-reconfigurable modular robotic system. IEEE/ASME Trans. Mechatronics 7 (4), 431-441.

Théraulaz, G., Bonabeau, E.W., 1995. Modelling the collective building of complex architectures in social insects with lattice swarms. J. Theor. Biol. 177, 381-400. 